\title{
AN EXPANSION WAVE IN THE NON-HOMENTROPIC FLOWS OF MARTIN AND LUDFORD
}

\author{
BY \\ J. A. STEKETEE \\ Delft University of Technology
}

1. Introduction. In 1954 Martin and Ludford published a paper [1] on the unsteady rectilinear motion of a perfect gas with non-uniform entropy distribution. The entropy distribution was chosen in such a way that the characteristic equations could be taken together to form an integrable combination, leading to generalized Riemann invariants.

As far as the author is aware, few applications of this type of flow have been discussed. In the original paper some simple examples are computed and it is pointed out that the lines of constant pressure differ from the lines of constant density.

In the present paper the expansion to vacuum of a Martin-Ludford gas is studied. The initial situation, however, is not a uniform gas at rest under constant pressure, but is a non-uniform gas exposed to a constant acceleration and moving as a rigid body, without compressions or expansions. This motion is generated by a pressure distribution, where equal elements of mass are exposed to equal pressure differences.

It is supposed that at some instant $t=\tau$, when the non-uniform gas cloud has a speed $u=U$, the pressure at the point of highest pressure is instantaneously reduced to zero. Rarefaction waves then begin to penetrate into the gas; the expansion to vacuum is pursued in detail. The problem may be described concisely as the generalization of the complete centered simple wave of the homentropic flow to the Martin-Ludford flows.

In Sec. 2 the basic equations of Martin and Ludford are obtained. The derivation differs from the original one, and an effort is made to present the theory simply and concisely. In Sec. 3 the flow, in which both the generalized Riemann invariants are constant, is considered. This is the fluw where the gas moves as a rigid body with constant acceleration. In Sec. 4 the generalized simple wave is considered. This part resembles closely the concise presentation of Martin and Ludford, but discusses also a slight variation showing some similarity with the methods used in homentropic flow. Some details about characteristics are also presented. In Sec. 5 the expansion to vacuum of the non-uniform gas is considered in the $p, h$-plane, $h$ being the Lagrangian coordinate. The answers are obtained in terms of these variables and a picture of the $p, h$-plane is drawn. In Sec. 6 the $h, t$-plane is considered in some detail, while in Sec. 7 the $x, t$-plane is considered.

2. Equations of motion, characteristics, Riemann invariants. We begin with the equations of motion in the Lagrangian form. These equations are well known and have been given by several authors $[2,3]$. They may be written in the form

$$
\begin{gathered}
(\partial V / \partial t)-(\partial u / \partial h)=0, \quad(\partial u / \partial t)+(\partial p / \partial h)=0 \\
p V^{\gamma}=B(h), \quad B(h)=\exp \left[\left(S-S_{0}\right) / c_{v}\right]
\end{gathered}
$$


with $t$ denoting the time, $h$ the Lagrangian coordinate defined by its differential, the mass increment $d h=\rho_{0} d x_{0}$ (position $x$ and density $\rho$ at time $t=0$ ), $u$ the velocity, $V$ the specific volume $\left(V=\rho^{-1}\right), S$ the entropy per unit mass, $S_{0}$ a constant of integration, $c_{\text {. }}$ the specific heat at constant volume, assumed constant, and $\gamma$ the constant ratio of the specific heats at constant pressure and constant volume $\left(\gamma=c_{p} c_{p}^{-1}\right)$.

It will be assumed that no shock waves occur and, since other effects of viscosity and heat conduction are also omitted, the fluid elements will retain their entropy for all time. Hence the entropy distribution $B$ depends on $h$ only.

Changing the independent variables in (2.1) and (2.2) from $h, t$ to $\xi(h, t), \eta(h, t)$, one can write these equations in the form of Jacobians as follows:

$$
\begin{aligned}
{[\partial(V, h) / \partial(\xi, \eta)]+[\partial(u, t) / \partial(\xi, \eta)] } & =0, \\
{[\partial(u, h) / \partial(\xi, \eta)]-[\partial(p, t) / \partial(\xi, \eta)] } & =0,
\end{aligned}
$$

provided the Jacobian $\mathcal{J}$,

$$
\mathscr{J}=\partial(h, t) / \partial(\xi, \eta)
$$

is different from zero.

Selecting $\xi=h, \eta=p$ in (2.5) and (2.6), one can write these equations in the form

$$
\begin{gathered}
(\partial u / \partial h)(\partial t / \partial p)-(\partial t / \partial h)(\partial u / \partial p)-(\partial V / \partial p)=0, \\
(\partial u / \partial p)-(\partial t / \partial h)=0 .
\end{gathered}
$$

It is easily verified that

$$
\partial V / \partial p=-V^{2} a^{-2}=-\gamma^{-1} B^{1 / \gamma} p^{-(\gamma+1) / \gamma},
$$

with $a$ denoting the local adiabatic speed of sound.

Eqs. (2.1) and (2.2) invite the introduction of "potentials" or "stream functions". Defining the functions $x$ and $K$ by

$$
\begin{aligned}
d x & =V d h+u d t, \quad d K=u d h-p d t, \\
\partial x / \partial h & =V, \quad \partial x / \partial t=u=\partial K / \partial h, \quad \partial K / \partial t=-p,
\end{aligned}
$$

it is clear that $x$ satisfies the continuity equation (2.1) identically, while the momentum equation (2.2) is identically satisfied by $K$. The function $x$ is the cartesian coordinate specifying the position of an element with label $h$ at time $t$. The function $K$ is connected with momentum. Considering $K$ as a function of $x$ and $t$ one is led, on using the relations (2.11), to the form

$$
d K=\rho u d x-\left(p+\rho u^{2}\right) d t,
$$

which identically satisfies the Eulerian momentum equation in conservation form.

In relation to Eqs. (2.8) and (2.9) it is convenient to introduce a function $M$ defined as a Legendre transformation of $K$ by

$$
\begin{aligned}
M & =K+p t, \quad d M=u d h+t d p, \\
\partial M / \partial h & =u, \quad \partial M / \partial p=t .
\end{aligned}
$$

Eq. (2.9) is identically satisfied by $M$ while (2.8) becomes the Monge-Ampère equation

$$
\left(\partial^{2} M / \partial p^{2}\right)\left(\partial^{2} M / \partial h^{2}\right)-\left(\partial^{2} M / \partial h \partial p\right)^{2}+V^{2} a^{-2}=0,
$$


as obtained by Martin and Ludford.

In order to study the characteristics of the system (2.8) and (2.9) or of Eq. (2.14), one may pose the Cauchy problem. In the first case initial data of $u$ and $t$ must be assigned along some initial curve. In the case of (2.14) in addition to $u$ and $t, M$ must also be assigned, while moreover the strip condition in (2.13) has to be satisfied along the initial curve. It is then easily found that two sets of characteristics exist. The one set has to satisfy the equations

$$
d t-(V / a) d h=0, \quad d u+(V / a) d p=0,
$$

while the other set satisfies

$$
d t+(V / a) d h=0, \quad d u-(V / a) d p=0 .
$$

Eqs. (2.15) and (2.16) are identical with the equations specifying the characteristics of the Lagrangian equations of motion (2.1) and (2.2). This is not surprising since the characteristics are essentially sound waves or mappings thereof. The only difference to be noted is that the first equations in (2.15) and (2.16) determine the slopes of the characteristics in the $h, t$-plane. The slopes of the characteristics in the $h, p$-plane are not so determined since $d p$ and $d h$ appear in different equations.

In the homentropic case, when the entropy distribution is uniform and $[B(h)]=$ constant, we have

$$
V a^{-1} d p=a \rho^{-1} d \rho=d(2 a /(\gamma-1)) .
$$

The second equations in (2.15) and (2.16) then lead, respectively, to the Riemann invariants $r$ and $s$, defined by

$$
r=u+2 a /(\gamma-1), \quad s=u-2 a /(\gamma-1) .
$$

These are constant along the $r$-characteristics defined by (2.15) and the $s$-characteristics defined by (2.16), respectively.

In the non-homentropic case $V a^{-1}$ does not depend only on $p$ but also on $h$ and a transformation as shown in (2.17) and (2.18) is in general impossible. Martin and Ludford noted, however, that by using the expression (2.13) for $d M$ in the direction of the characteristics the following can be done. Writing $d M=d(u h+t p)-h d u-p d t$, and substituting (2.15), one is led to

$$
d M=d(u h+t p)+h^{2}(V / a) d(p / h) .
$$

Selecting $B(h)$ in (2.3) and (2.4) to be of the form

$$
B(h)=C^{2 \gamma} h^{-3 \gamma+1},
$$

with $C$ an arbitrary constant, the combination $h^{2} V a^{-1}$ can be made to depend on $p h^{-1}$ alone and (2.19) may be rewritten as an exact differential, leading to the generalized Riemann invariant

$$
M-u h-t p-[(2 C \sqrt{\gamma}) /(\gamma-1)](p / h)^{(\gamma-1) / 2 \gamma}=r,
$$

which is constant along a characteristic of the family determined by (2.15). In analogy with the homentropic case they will be called $r$-characteristics. In the same way one finds from (2.16) that

$$
M-u h-t p+[(2 C \sqrt{\gamma}) /(\gamma-1)](p / h)^{(\gamma-1) / 2 \gamma}=s
$$


is constant along a characteristic of the family defined by (2.16) which are to be called $s$-characteristics. It is easily found from

$$
p V^{\gamma}=B(h)=C^{2 \gamma} h^{-(3 \gamma-1)}
$$

that

$$
a=C \sqrt{\gamma} / h^{-1}(p / h)^{(\gamma-1) / 2 \gamma} \quad V / a=(C / \sqrt{\gamma}) h^{-2}(h / p)^{(\gamma+1) / 2 \gamma}
$$

Substitution of the speed of sound $a$ and (2.13) in (2.21) and (2.22) allows these expressions to be rewritten in the form

$$
K-h\left(u+\frac{2 a}{\gamma-1}\right)=r, \quad K-h\left(u-\frac{2 a}{\gamma-1}\right)=s .
$$

3. The flow with $r=$ const and $s=$ const. Consider a flow in which the generalized Riemann invariants $r$ and $s$ defined by (2.21) and (2.22) are constant throughout. It follows that in such a flow

$$
M-u h-t p=\alpha_{0} . \quad p=\beta_{0} h,
$$

with $\alpha_{0}$ and $\beta_{0}$ constant. Expressions for $\alpha_{0}$ and $\beta_{0}$ in terms of $r, s, C$ and $\gamma$ may easily be found. The image of this flow in the $p, h$-plane is clearly a straight line through the origin. Taking differentials of the expressions in (3.1), one is led to

$$
d u=-\beta_{0} d t, \quad d p=\beta_{0} d h .
$$

Since $h$ essentially represents the mass of the gas, measured with respect to some suitably chosen origin, it is clear from the second relations in (3.1) and (3.2) that equal elements of mass are exposed to equal pressure differences. It follows from first principles of mechanics that all mass elements possess the same constant acceleration. This result is essentially contained in the first equation of (3.2).

The pressure in a mass element is entirely determined by its Lagrangian coordinate $h$, and other parameters such as time do not affect it. The same holds true for such other properties as the specific volume $V$, temperature $T$, and speed of sound $a$. One easily deduces from (2.23), (2.24) and the ideal gas law that

$$
V h^{3}=\text { const }, \quad a h=\text { const }, \quad T h^{2}=\text { const. }
$$

Expressions for the constants in terms of $\beta_{0}, C$ and the gas constant $R$ can be easily obtained. Typical differences between the gas considered here and a homentropic gas should be noted. While in a homentropic gas vanishing pressure implies vanishing density, temperature and speed of sound, it follows from (3.1) and (3.3) that, owing to the special entropy distribution (or heat content), for $h \rightarrow 0$ we have

$$
p \rightarrow 0, \quad V \rightarrow \infty, \quad \rho \rightarrow 0, \quad a \rightarrow \infty, \quad T \rightarrow \infty .
$$

The relations (3.3) imply that the pressure forces are engaged in accelerating the mass elements, but do not produce compressions or expansions since all fluid elements experience the same acceleration; the entire non-uniform mass of gas is moving as a rigid body with uniform acceleration or deceleration. It will be clear that initially a uniform velocity must exist throughout the gas to make this flow possible. Also, while it is not difficult to imagine this kind of flow, it is not immediately obvious where it may occur or how to produce it. 
It is of interest to consider some further details. From (3.3) the density as function of position $x$ at some instant and the connection between $h$ and $x$ at that instant can be calculated. Using the definition of $d h$ and the explicit value of the constant in the first relation of (3.3), one finds

$$
d x=C^{2} \beta_{0}^{-1 / \gamma} h^{-3} d h=\frac{1}{3} \beta_{0}^{-1 / 3 \gamma} C^{2 / 3} \rho^{-5 / 3} d \rho .
$$

A first integration shows that the natural choice for the origin of $h$ coincides with $x=-\infty$. If at the instant considered the gas extends along the negative $x$-axis over the interval $-\infty<x \leq 0$ while the total mass of the gas is $H$, one finds

$$
x=-\left(C^{2} / 2\right) \beta_{0}^{-1 / \gamma}\left(h^{-2}-H^{-2}\right) .
$$

Writing

$$
x_{0}=\left(C^{2} / 2\right) \beta_{0}^{-1 / \gamma} H^{-2},
$$

one also finds

$$
h=(C / \sqrt{2}) \beta_{0}^{-1 / 2 \gamma}\left(x_{0}-x\right)^{-1 / 2}, \quad \rho=(C / 2 \sqrt{2}) \beta_{0}^{-1 / 2 \gamma}\left(x_{0}-x\right)^{-3 / 2} .
$$

Clearly, for $x \rightarrow-\infty, \rho \rightarrow 0$ and $h \rightarrow 0$.

Considering this motion in the $h, t$-plane, it is easy to calculate the characteristics. From (2.15), (2.24) and (3.1) it follows that the $r$-characteristics in the $h, t$-plane satisfy

$$
d t-(C / \sqrt{\gamma}) \beta_{0}^{-(\gamma+1) / 2 \gamma} h^{-2} d h=0, \quad d u+(C / \sqrt{\gamma}) \beta_{0}^{(3 \gamma-1) / 2 \gamma} p^{-2} d p=0 .
$$

Integration gives

$$
\left(t-t_{0}\right) h=-(C / \sqrt{\gamma}) \beta_{0}^{-(\gamma+1) / 2 \gamma} \quad\left(u-u_{0}\right) p=(C / \sqrt{\gamma}) \beta_{0}^{(3 \gamma-1) / 2 \gamma}
$$

with $t_{0}$ and $u_{0}$ denoting integration constants, varying from one $r$-characteristic to another. It is clear that the $r$-characteristics in the $h, t$-plane are a family of equilateral hyperbolae (parameter $t_{0}$ ), with $h=0$ and $t=t_{0}$ as asymptotes. Since $0 \leq h \leq H$ in the region of interest, all the $r$-characteristics start at $t=-\infty$ and would (if $h$ extended that far) reach $h=+\infty$ for $t=t_{0}$. The second expression in (3.10), which may be written

$$
\left(u-u_{0}\right) h=(C / \sqrt{\gamma}) \beta_{0}^{(\gamma-1) / 2 \gamma},
$$

gives the value of $u$ along the characteristic, once the value of the parameter $u_{0}$ is assigned.

In the same way one finds for the $s$-characteristics

$$
\left(t-t_{0}\right) h=(C / \sqrt{\gamma}) \beta_{0}^{-(\gamma+1) / 2 \gamma} \quad\left(u-u_{0}\right) h=-(C / \sqrt{\gamma}) \beta_{0}^{(\gamma-1) / 2 \gamma} .
$$

These hyperbolae would start at $h=\infty$ for $t=t_{0}$ and reach $h=0$ for $t=+\infty$.

In order to specify the parameters $u_{0}$ and $t_{0}$ completely, it is sufficient to specify the situation at one instant. If the mass of gas has the velocity $u=U$ at the instant $t=\tau$ one finds

$$
t_{0}=\tau \pm(C / \sqrt{\gamma}) \beta_{0}^{-(\gamma+1) / 2 \gamma}\left(1 / h_{\tau}\right), \quad u_{0}=U \mp(C / \sqrt{\gamma}) \beta_{0}^{(\gamma-1) / 2 \gamma}\left(1 / h_{\tau}\right),
$$

where the top signs apply to the $r$-characteristics and the lower signs to the $s$-characteristics. The expressions (3.13) assign to each value $h_{\tau}\left(0 \leq h_{\tau} \leq H\right)$ on the line $t=\tau$ in the $h, t$-plane one $r$-characteristic and one $s$-characteristic together with the appropriate values of $u_{0}$. The velocity at another point of the $r$ - and $s$-characteristics is then given by (3.11) and (3.12), respectively. 
The flow considered here is singular and degenerate in a mathematical sense. The coordinates $p$ and $h$ are not independent, the determinant (2.7) vanishes, $u$ and $t$ are not single-valued functions of $p$ and $h$ and the one-one mapping from the $p, h$-plane to the $h, t$-plane has broken down. A more elaborate discussion of flows with $p=p(h)$ has been given by Weir [4] where the properties discussed here and some others are obtained.

4. The flow regime with $r=r_{0}=$ const. A flow of this kind may be considered as the generalization of the simple wave in homentropic flow. Throughout the flow we have

$$
M-u h-t p-[2 C \sqrt{\gamma} /(\gamma-1)](p / h)^{(\gamma-1) / 2 \gamma}=r_{0}=\text { const, }
$$

Along an $s$-characteristic the expression (2.22) is constant; together with (4.1) this leads to

$$
M-u h-t p=\alpha, \quad p=\beta h,
$$

with $\alpha$ and $\beta$ constant. Clearly, the relations obtained in the preceding section for the entire flow regime now apply along an $s$-characteristic. As one changes from one $s$-characteristic to another the values of $\alpha$ and $\beta$ change.

The second relation in (4.2) indicates that the $s$-characteristics in the $p, h$-plane form a family of straight lines through the origin; hence the wave is centered in the $p, h$-plane.

Differentiation of the first expression in (4.2) along the characteristic leads to

$$
d u+\beta d t=0,
$$

indicating that along an $s$-characteristic $u$ decreases $(\beta>0)$ or increases $(\beta<0)$ proportionally to $t$ (compare (3.2)). It also follows that now along an $s$-characteristic relations (3.3) apply.

In order to obtain expressions for $u$ and $t$ in the simple wave case we return, following Martin and Ludford, to (4.1). This expression is a first-order partial differential equation for $M$ and can be written in the form

$$
h(\partial M / \partial h)+p(\partial M / \partial p)=M-[2 C \sqrt{\gamma} /(\gamma-1)](p / h)^{(\gamma-1) / 2 \gamma}-r_{0} .
$$

Two independent integrals of the associated system

$$
d h / h=d p / p=d M /\left\{M-[2 C \sqrt{\gamma} /(\gamma-1)](p / h)^{(\gamma-1) / 2 \gamma}-r_{0}\right\},
$$

are easily found and the general solution of (4.4) then takes the form

$$
M=r_{0}+[2 C \sqrt{\gamma} /(\gamma-1)](p / h)^{(\gamma-1) / 2 \gamma}+h F(p / h),
$$

with $F$ representing an arbitrary function of $p h^{-1}$. Differentiation of $M$ with respect to $p$ and $h$ then leads to $t$ and $u$, respectively. One finds

$$
\begin{gathered}
t=\partial M / \partial p=F^{\prime}(p / h)+\left(C /(\sqrt{\gamma}) p^{-(\gamma+1) / 2 \gamma} h^{-(\gamma-1) / 2 \gamma)},\right. \\
u=\partial M / \partial h=F(p / h)-(p / h) F^{\prime}(p / h)-(C / \sqrt{\gamma}) p^{(\gamma-1) / 2 \gamma} h^{-(3 \gamma-1) / 2 \gamma)},
\end{gathered}
$$

with the primes denoting differentiation w. r. t. $p h^{-1}$. By employing the relations (2.25) the expressions for $t$ and $u$ may be put in the form

$$
\begin{gathered}
t-(V / a) h=F^{\prime}(p / h), \\
u+(1 / \gamma) a=F(p / h)-(p / h) F^{\prime}(p / h) .
\end{gathered}
$$


An alternative method of determining $t$ and $u$ is to employ the equations (2.16) for the $s$-characteristics. Since $p h^{-1}$ is constant along an $s$-characteristic these expressions can be easily integrated. Two arbitrary functions of $p h^{-1}$ are introduced in this process as integration constants, one in the expression for $t$, the other in the expression for $u$. These two functions, however, are not independent; substitution of $u$ and $t$ in the first and second equation of the set (2.15) should result in the same slope for the $r$-characteristic in the $p, h$-plane. This leads to the condition

$$
\left|\begin{array}{ll}
\frac{\partial t}{\partial p} & \frac{\partial t}{\partial h}-\frac{V}{a} \\
\frac{\partial u}{\partial p}+\frac{V}{a} & \frac{\partial u}{\partial h}
\end{array}\right|=0
$$

representing a relation between the two functions of $p h^{-1}$. When this is worked out the relations for $F$ appearing in (4.7) and (4.8) can be retrieved.

With the calculation of $t$ and $u$ the generalized simple wave flow is essentially complete.

As a further point we consider the form of the $r$-characteristics. These are determined by Eqs. (2.15). Substituting (4.7) in the first equation of (2.15), one finds

$$
\begin{aligned}
& F^{\prime \prime}(\beta) d \beta-\frac{C}{\sqrt{\gamma}} \\
& \quad \cdot\left\{\frac{\gamma+1}{2 \gamma} p^{-(3 \gamma+1) / 2 \gamma} h^{-(\gamma-1) / 2 \gamma} d p+\frac{3 \gamma-1}{2 \gamma} p^{-(\gamma+1) / 2 \gamma} h^{-(3 \gamma-1) / 2 \gamma} d h\right\}=0,
\end{aligned}
$$

with $\beta=p h^{-1}$. The expression in braces is not an exact differential, but may be made into one by multiplication with a suitable power of $\beta$. Multiplying (4.12) with $\beta^{n}$, one finds that with

$$
n=(\gamma+1) / 4 \gamma,
$$

one can rewrite the expression in the form

$$
F^{\prime \prime}(\beta) \beta^{n} d \beta+(2 C / \sqrt{\gamma}) d\left(h^{-1} \beta^{-n}\right)=0 .
$$

The integration leading to the form of the $r$-characteristics is then easily achieved once $F(\beta)$ is known.

The generalized simple wave with $s=s_{0}=$ const can be studied in the same way. The formulae obtained in that case are, up to some plus and minus signs, identical with the relations obtained in this section.

5. A special generalized simple wave. In order to apply the relations of the preceding section the following problem will be considered. A body of gas, say a gas cloud, with total mass $H$, filling up a halfspace $-\infty \leq x \leq 0$ at each moment and satisfying the relations of Sec. 3 is exposed to a uniform acceleration under the influence of the pressure distribution

$$
p=\beta_{0} h=\left(p_{0} / H\right) h .
$$

At time $t=\tau$ this body of gas has acquired the velocity $U$. Since the acceleration with $\beta_{0}>0$ is in the direction of decreasing $h$ and $x, U$ will usually be negative. In the $p, h-$ plane the motion is represented by the segment of the straight line (5.1) in the interval $0 \leq h \leq H$. (See $O A$ in Fig. 1.) 
At the instant $t=\tau$ the pressure at the extremity $h=H$ suddenly drops from the value $p=p_{0}$ to $p=0$. The resulting expansion of the gas cloud is to be considered.

For $t<\tau$ the motion is of the kind $r=r_{0}=$ const, $s=s_{0}=$ const. and since only sound waves from the right will penetrate into the gas along $s$-characteristics, it may be expected that the function $r=r_{0}=$ const will retain its original value throughout the expansion. The flow for $t>\tau$ is then of the type discussed in Sec. 4 and the relations obtained there immediately apply.

In order to obtain the correct boundary conditions for the determination of $F(\beta)$ in (4.7) and (4.8) the following points have to be noted.

At time $t=\tau$ the pressure at $h=H$ instantaneously drops to zero; hence along $A B$ in Fig. $1 t=\tau$. Since $F^{\prime}$ depends on $\beta=p h^{-1}$ only, substitution of this condition into (4.7) leads to

$$
F^{\prime}(\beta)=\tau-(C / \sqrt{\gamma})(1 / H) \beta^{-(\gamma+1) / 2 \gamma},
$$

and with this expression for $F^{\prime}(\beta), t$ now becomes

$$
t=\tau+(C / \sqrt{\gamma}) \beta^{-(\gamma+1) / 2 \gamma}(1 / h-1 / H),
$$

In order to verify this expression the first sound wave travelling into the gas may be checked. Along this sound wave we have, from (2.16), (2.24) and (5.1),

$$
d t+(C / \sqrt{\gamma}) \beta_{0}^{-(\gamma+1) / 2 \gamma}\left(d h / h^{2}\right)=0 .
$$

Integration of (5.4) with the initial condition $h=H$ for $t=\tau$ then leads to the answer obtained from (5.3) upon substitution of $\beta=\beta_{0}$.

Integration of (5.2) gives

$$
F(\beta)=\tau \beta-[2 C \sqrt{\gamma} /(\gamma-1)](1 / H) \beta^{(\gamma-1) / 2 \gamma}+F(0),
$$

with $F(0)$ an integration constant. Substitution in (4.8) gives

$$
u=F(0)-[(\gamma+1) /(\gamma-1)](C / \sqrt{\gamma}) \beta^{(\gamma-1) / 2 \gamma}(1 / H)-(C / \sqrt{\gamma}) \beta^{(\gamma-1) / 2 \gamma}(1 / h) .
$$

When the expansion process begins at time $t=\tau$ the velocity is $u=U$. This is particularly the case at $h=H, p=p_{0}$. Substituting these values in (5.6) gives

$$
\left.F(0)=U+[2 \gamma /(\gamma-1)](C / \sqrt{\gamma}) \beta_{0}^{(\gamma-1) / 2 \gamma} 1 / H\right),
$$

and leads finally to

$$
\begin{aligned}
u=U+ & (2 \gamma /(\gamma-1))(C / \sqrt{\gamma}) \beta_{0}^{(\gamma-1) / 2 \gamma}(1 / H) \\
& -((\gamma+1) /(\gamma-1))(C / \sqrt{\gamma}) \beta^{(\gamma-1) / 2 \gamma}(1 / H)-(C / \sqrt{ } \bar{\gamma}) \beta^{(\gamma-1) / 2 \gamma}(1 / h)
\end{aligned}
$$

In order to verify this expression the first sound wave can again'be checked. From (2.16), (2.24) and (5.1) it follows that along this $s$-characteristic

$$
d u-(C / \sqrt{\gamma}) \beta_{0}^{-(\gamma+1) / 2 \gamma} h^{-2} d p=d u-(C / \sqrt{\gamma}) \beta_{0}^{(\gamma-1) / 2 \gamma} h^{-2} d h=0 .
$$

Integration of (5.9) with the initial condition $u=U$ for $h=H$ then gives

$$
u=U+(C / \sqrt{\gamma}) \beta_{0}^{(\gamma-1) / 2 \gamma}(1 / H)-(C / \sqrt{\gamma}) \beta_{0}^{(\gamma-1) / 2 \gamma}(1 / h) .
$$

This answer can be obtained immediately from (5.8) upon substituting $\beta=\beta_{0}$, the value of $\beta$ for the first $s$-characteristic. To clarify relation (5.10) it is sufficient to realize that up to the arrival of the first sound wave the gas is exposed to the pressure distri- 
bution (5.1) and the acceleration $-\beta_{0}$. If at the instant $t$ the first sound wave has reached a position $h$ determined from (5.3) with $\beta=\beta_{0}$ the velocity at that point is no longer $U$, but

$$
u=U-\beta_{0}(t-\tau),
$$

leading to (5.10).

The relations for $t$ and $u$ can be put in several alternative forms upon using (2.24). So one can write

$$
\begin{aligned}
t-\tau & =(V / a) h(1-(h / H)), \\
u-U & =(2 /(\gamma-1)) a_{A}-(1 / \gamma) a[1+((\gamma+1) /(\gamma-1))(h / H)],
\end{aligned}
$$

with $a_{A}$ denoting the speed of sound in $A$ with $p=p_{0}, h=H$.

It is seen from (5.3) that the first sound wave reaches $h=0$ after an infinite time. According to (5.10) the velocity there is then $u=-\infty$, since the gas has been exposed to the acceleration $-\beta_{0}$ during an infinite time.

For the particle $h=H$ the pressure drop from $p=p_{0}$ to $p=0$ is achieved instantaneously. The relation (5.13) gives for $h=H$

$$
u+(2 /(\gamma-1)) a=U+(2 /(\gamma-1)) a_{A} .
$$

It follows from (2.24) that for fluid elements $h>0$ the speed of sound approaches zero with $p \rightarrow 0$ and so at $h=H$ the velocity increases instantaneously from $U$ to $U+$ $(2 /(\gamma-1)) a_{A}$. The relation (5.14) and this velocity increase are the same as in homentropic flow.

It is easily checked from (5.3) that for particles in the range $0<h<H$ the pressure $p=0$ is reached after an infinite time. The terminal velocity of these fluid elements is also

$$
u=U+(2 /(\gamma-1)) a_{A} .
$$

This is a remarkable result, since at the moment that the first sound wave arrives at $h$ $(<H)$ the velocity has decreased from the value $U$, due to the action of the acceleration $-\beta_{0}$, while the maximum speed of sound at that location exceeds $a_{A}$ by a factor $H h^{-1}$.

Some further details of the flow in the $p, h$-plane may be considered. The distance travelled by a fluid element can be written in the form

$$
x\left(t_{2}\right)-x\left(t_{1}\right)=\int_{t_{1}}^{t_{2}} u d t=\int_{\beta_{1}}^{\beta_{2}} u(\beta) t^{\prime}(\beta) d \beta,
$$

where $\beta$ has to be considered with $h$ fixed. Introducing the abbreviations

$$
\begin{aligned}
& P_{1}=U+(2 C \sqrt{\gamma} /(\gamma-1))\left(p_{0} / H\right)^{(\gamma-1) / 2 \gamma}(1 / H), \\
& P_{2}=(C / \sqrt{\gamma})(((\gamma+1) /(\gamma-1))(1 / H)+(1 / h)), \\
& P_{3}=((\gamma+1) / 2 \gamma)(C / \sqrt{\gamma})((1 / h)-(1 / H)),
\end{aligned}
$$

one may rewrite this integral in the form

$$
x\left(t_{2}\right)-x\left(t_{1}\right)=-\int_{\beta_{1}}^{\beta_{2}}\left(P_{1}-P_{2} \beta^{(\gamma-1) / 2 \gamma}\right) P_{3} \beta^{-(3 \gamma+1) / 2 \gamma} d \beta
$$

which is easily evaluated. 
It is found from (5.2) that

$$
F^{\prime \prime}(\beta)=((\gamma+1) / 2 \gamma)(C / \sqrt{\gamma}) \beta^{-(3 \gamma+1) / 2 \gamma}(1 / H),
$$

and Eq. (4.14) for the $r$-characteristics can be written

$$
n \beta^{-(n+1)} H^{-1} d \beta+d\left(h^{-1} \beta^{-n}\right)=0,
$$

with $n$ given by (4.13). Integration leads to the form

$$
(h / p)^{n}\left(h^{-1}-H^{-1}\right)=R=\text { const, }
$$

with $R$ denoting the parameter of the family.

Considering the intersection of the $r$-characteristics with the $s$-characteristic $p=\beta_{0} h$, one notes that the values of $R$ range from $R=\infty$ for the characteristic intersecting at $h=0$ to $R=0$ for the characteristic intersecting at $h=H$. Further inspection shows that the $r$-characteristics with $R>0$ all pass through $p=0, h=H$. The slope of the $r$-characteristics is found to be

$$
R(d p / d h)=-(p / h)^{1-n}(((1-n) / n)(1 / h)+(1 / H)) .
$$

It shows that for the characteristics with $R \neq 0, d p / d h=0$ for $p=0$ and the $r$-characteristics are tangent to the $p$-axis in $p=0, h=H$.

A qualitative sketch of the flow in the $p, h$-plane is shown in Fig. 1.

6. The flow in the $(h, t)$-plane. The expressions obtained in Sec. 5 are sufficiently simple that a switch to the $h, t$-plane can be made without difficulty.

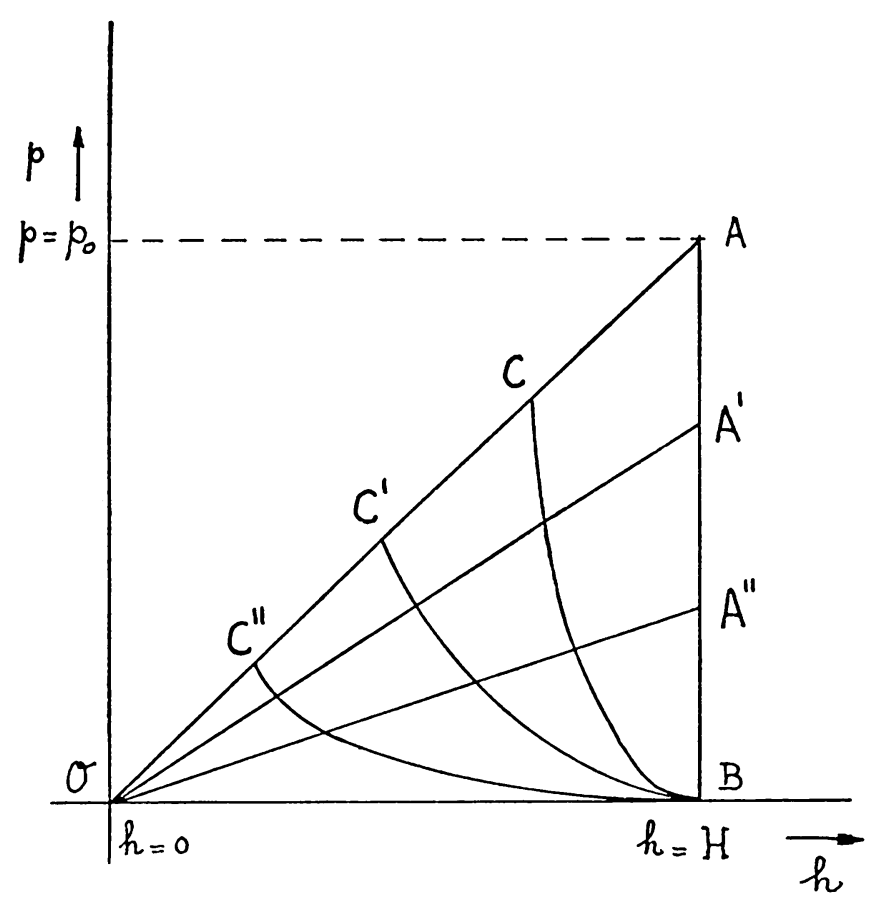

Frg. 1. $O A$ is $p=\beta_{0} h ; O A, O A^{\prime}, O A^{\prime \prime}$ are $s$-characteristics; $B C, B C^{\prime}, B C^{\prime \prime}$ are $r$-characteristics. Along $A B, t=\tau$. 
From (5.3) one easily deduces

$$
(p / h)^{(\gamma+1) / 2 \gamma}=(C / \sqrt{\gamma})[(H-h) /(H h(t-\tau))],
$$

giving $p$ in terms of $h$ and $t$. Using (6.1) in (5.8), (2.23) and (2.24) one obtains

$$
\begin{gathered}
u=\left(U+\frac{2}{\gamma-1} a_{A}\right)-\frac{C}{\sqrt{\gamma}} \cdot \frac{(\gamma-1) H+(\gamma+1) h}{(\gamma-1) h H} \cdot\left\{\frac{C}{\sqrt{\gamma}} \frac{H-h}{H h(t-\tau)}\right\}^{(\gamma-1) /(\gamma+1)}, \\
V=\left(C^{2} / h^{3}\right)\{(C / \sqrt{\gamma})((H-h) / H h(t-\tau))\}^{-2 /(\gamma+1)} \\
a=(C \sqrt{\gamma} / h)\{(C / \sqrt{\gamma})((H-h) / H h(t-\tau))\}^{(\gamma-1) /(\gamma+1)} \\
V / a=H(t-\tau) / h(H-h) .
\end{gathered}
$$

The differential equations (2.15) and (2.16) for the $r$-and $s$-characteristics in the $h$, $t$-plane are then

$$
d t \pm(H(t-\tau) / h(H-h)) d h=0,
$$

and can be easily integrated. An alternative way to find the characteristics in the $h, t$-plane is as follows. Along the $s$-characteristics $p / h=$ const and so from (6.1)

$$
h(t-\tau) /(H-h)=\text { const. }
$$

The $r$-characteristics in the $p, h$-plane are given by (5.21), and using (6.1) one obtains in the $h, t$-plane

$$
((H-h)(t-\tau)) / h=\text { const. }
$$

Taking $S^{*}$ and $R^{*}$ as the values of the constants in (6.7) respectively (6.8) one may rewrite these equations in the form

$$
h\left(t+S^{*}-\tau\right)=H S^{*}, \quad(H-h)\left(t+R^{*}-\tau\right)=H R^{*},
$$

indicating that in the $h, t$-plane the characteristics are two families of equilateral hyperbolae. The $s$-characteristics have the asymptotes $h=0, t=\tau-S^{*}$ while the $r$-characteristics have the asymptotes $h=H, t=\tau-R^{*}$. With a change of $R^{*}$ and $S^{*}$ not only do the asymptotes shift but the lengths of the axes of the hyperbolae also change, due to the appearance of $R^{*}$ and $S^{*}$ on the right-hand side in (6.9) and (6.10). This is different from the characteristics considered in Sec. 3 .

It is noticed from (6.9) that all the $s$-characteristics pass through $h=H, t=\tau$, while (6.10) shows that all the $r$-characteristics in the expansion are parts of hyperbolae passing through $h=0, t=\tau$. The expansion begins with the first sound wave, the $s$-characteristic through $h=H$ of the family of $s$-characteristics discussed in Sec. 3 . From (3.13) and (3.12) it follows that this characteristic has the equation

$$
\left(t-\tau+\frac{C}{\sqrt{\gamma}} \beta_{0}^{-(\gamma+1) / 2 \gamma} \frac{1}{H}\right) h=\frac{C}{\sqrt{\gamma}} \beta_{0}^{-(\gamma+1) / 2 \gamma} .
$$

Since it is also the first characteristic in the expansion, it is included in (6.9). Comparison shows that

$$
S^{*}=\left(C /(\sqrt{\gamma}) \beta_{0}^{-(\gamma+1) / 2 \gamma}(1 / H)=\left(V_{A} / a_{A}\right) H .\right.
$$

For the other s-characteristics in the expansion region one finds, comparing it with (6.1),

$$
S^{*}=(C / \sqrt{\gamma}) \beta^{-(\gamma+1) / 2 \gamma}(1 / H) .
$$


Since $\beta<\beta_{0}$, the values of $S^{*}$ increase and the axes of the hyperbolae increase in length. At the same time the horizontal asymptotes shift in the negative $t$-direction and the parts of the hyperbolae in the expansion fan are to the right of the hyperbola (6.11). A qualitative sketch of the $h, t$-plane is shown in Fig. 2.

There are two kinds of $r$-characteristics in the $h, t$-plane. In the first place there are $r$-characteristics of the type discussed in Sec. 3 which apply until the first sound wave, initiating the expansion, arrives. Secondly, there are the $r$-characteristics in the expansion

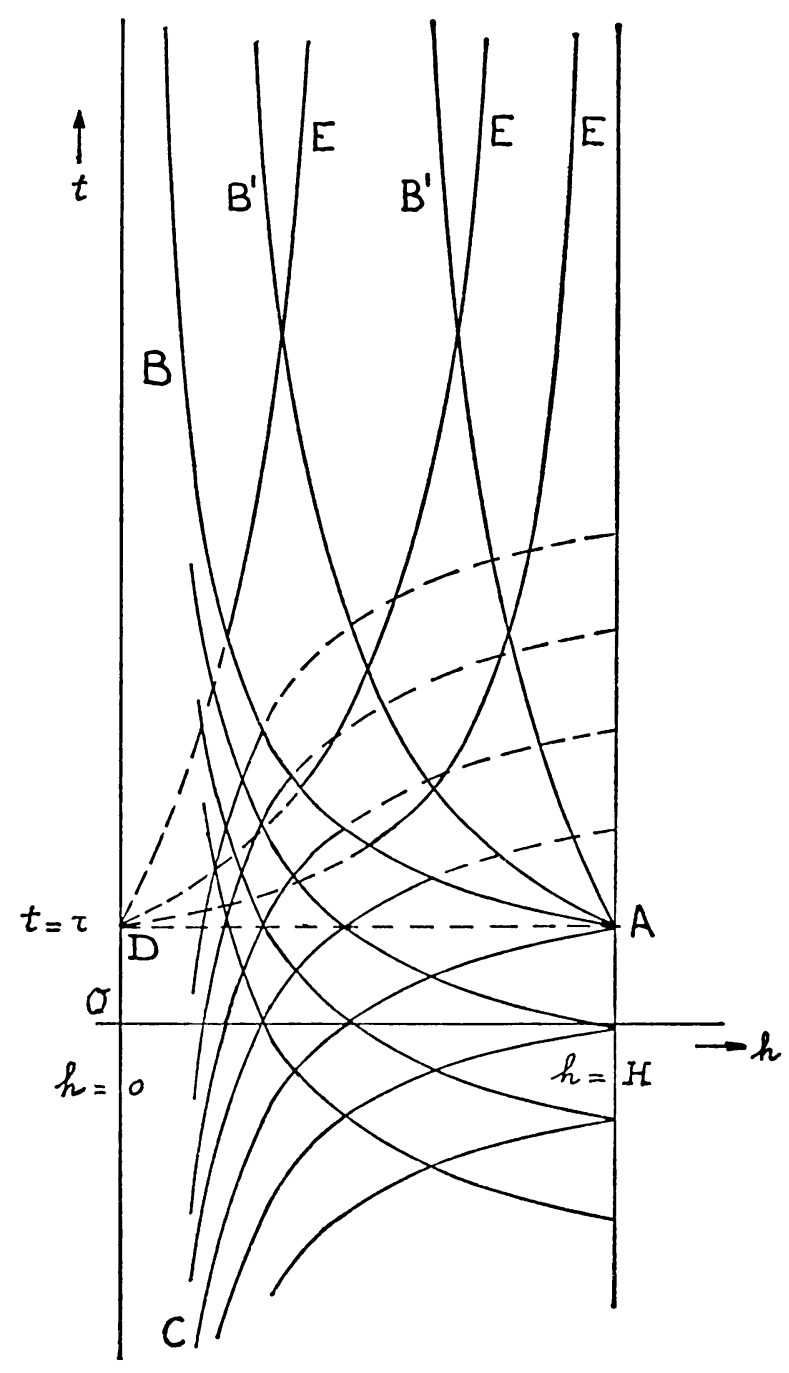

FIG. 2. $A B$ is the first sound wave, with the expansion fan on the right and the constant acceleration regime on the left. The $s$-characteristics in the expansion region are the curves $A B^{\prime}$. The $r$-characteristics are indicated by $D E$; only the solid parts, to the right of $A B$, have physical significance. The curves obtained from $A B$ by translation downwards are $s$-characteristics in the constant acceleration regime. $A C$ and the curves obtained from it by the translation in the $t$-direction are $r$-characteristics. Only the solid parts to the left of $A B$ have physical significance. The particle paths are lines $h=$ const. and have not been drawn. 
region. In order to match the two sets of characteristics across the first sound wave, it is necessary to know where they intersect the first sound wave.

The $r$-characteristic of Sec. 3 passing through $h_{\tau}$ at time $t=\tau$ can be written, from (3.10) and (3.13), in the form

$$
t-\tau=(C / \sqrt{\gamma}) \beta_{0}^{-(\gamma+1) / 2 \gamma}\left(\left(1 / h_{\tau}\right)-(1 / h)\right) .
$$

This characteristic intersects the first sound wave (6.11) at the position $h$, and time $t$, determined by

$$
h^{-1}=\frac{1}{2}\left(H^{-1}+h_{\tau}^{-1}\right), \quad t-\tau=(C / 2 \sqrt{\gamma}) \beta_{0}^{-(\gamma+1) / 2 \gamma}\left(h_{\tau}^{-1}-H^{-1}\right) .
$$

Eq. (6.10) for the $r$-characteristics in the expansion region can be written in the form

$$
(t-\tau)((1 / h)-(1 / H))=R^{*} / H .
$$

For the $r$-characteristic passing through the intersection point $h, t$, determined by (6.15) and $(6.16)$, we then find

$$
R^{*} / H=(C / 4 \sqrt{\gamma}) \beta_{0}^{-(\gamma+1) / 2 \gamma}\left(\left(1 / h_{\tau}\right)-(1 / H)\right)^{2} .
$$

This expresses $R^{*}$ in terms of known constants; $h_{\tau}$ denotes the point on the line $t=\tau$ where the characteristic $(6.14)$ originates.

7. The flow in the $x, t$-plane. Finally, also the physical plane, the $x, t$-plane, should be considered. In the $x, t$-plane the flow is built up of two regions, similar to the situation in $h, t$-plane. First there is the region with uniform acceleration, as discussed in Sec. 3; secondly, there is the expansion region, discussed in Sec. 5. The two regions are separated by the first sound wave. In the uniform acceleration region the velocity $u$, satisfying the initial condition at time $t=\tau$, is given by

$$
u=U-\beta_{0}(t-\tau),
$$

and, as discussed in Sec. 3, is independent of $h$. The specific volume $V$ is given by (3.3); putting in the values of the constant, one obtains

$$
V=\left(C^{2} / h^{3}\right)\left(\beta_{0}\right)^{-1 / \gamma}
$$

The clue to the conversion from the $h, t$-plane to the $x, t$-plane is the relation in (2.11):

$$
d x=V d h+u d t .
$$

Substitution of (7.1) and (7.2) leads upon integration to

$$
x=U(t-\tau)-\left(\beta_{0} / 2\right)(t-\tau)^{2}-\left(C^{2} / 2\right) \beta_{0}^{-1 / \gamma}\left(\left(1 / h^{2}\right)-\left(1 / H^{2}\right)\right) .
$$

The integration constant appearing in the solution has been chosen in such a way that at $t=\tau, x=-\infty$ for $h=0$ and $x=0$ for $h=H$. For $t=\tau$ the relation (7.4) is clearly identical with (3.6).

The particle paths, curves with $h=$ const, are clearly parabolae. The characteristics in the uniform acceleration region are calculated in Sec. 3 in terms of $h$ and $t$. Eliminating $h$ between these relations and (7.4) results in expressions for the characteristics in terms of $x$ and $t$. Omitting the details, one finds

$$
\begin{aligned}
x=-\left(C^{2} / 2\right) \beta_{0}^{-1 / \gamma} & \left(h_{\tau}^{-2}-H^{-2}\right) \\
& +\left(U \pm\left(C \sqrt{\gamma} / h_{\tau}\right) \beta_{0}^{(\gamma-1) / 2 \gamma}\right)(t-\tau)-((\gamma+1) / 2) \beta_{0}(t-\tau)^{2},
\end{aligned}
$$


where the plus sign applies to the $r$-characteristics, the minus sign to the $s$-characteristics and $h_{\tau}$ represents the parameter.

It is clear that the $r$ - and $s$-characteristics are again parabolae. The $s$-characteristic separating the expansion region from the uniform acceleration region passes through $h=H$ at $t=\tau$ and has the equation

$$
x=\left(U-a_{A}\right)(t-\tau)-((\gamma+1) / 2) \beta_{0}(t-\tau)^{2} .
$$

The same steps have to be taken with the formulae of the expansion region in order to map it in the $x, t$-plane. The velocity $u$ and the specific volume $V$ are given by (6.2) and (6.3). Considering then the relation (7.3) the integration to the time is easily achieved. The choice of the integration constant is such that $h=H, t=\tau$ has to coincide with $x=0, t=\tau$; finally one finds

$$
\begin{gathered}
x=\left(U+2 a_{A} /(\gamma-1)\right)(t-\tau)-((\gamma+1) / 2)(C / \sqrt{\gamma})\left(h^{-1}+((\gamma+1) /(\gamma-1)) H^{-1}\right) \\
\cdot((C / \sqrt{\gamma})((H-h) /(H h(t-\tau))))^{(\gamma-1) /(\gamma+1)}(t-\tau) .
\end{gathered}
$$

The form of the particle paths, lines with $h=$ const, can be easily read off from (7.7). In particular, for $h=H$, the second term in (7.7) vanishes and this vacuum edge of the expansion wave moves with the constant speed $U+(2 /(\gamma-1)) a_{A}$.

The $s$-characteristics are the lines $p h^{-1}=\beta$, and from (6.1) and (6.7) it is clear that the factor with the exponent $(\gamma-1) /(\gamma+1)$ in $(7.7)$ reduces to a constant. By writing the $s$-characteristics in the form

$$
(C / \sqrt{\gamma})((1 / h)-(1 / H))=\beta^{(\gamma+1) / 2 \gamma}(t-\tau),
$$

the term $1 / h$ in (7.7) can also be eliminated and finally one obtains for the $s$-characteristics

$$
\begin{array}{r}
x=\left(U+2 a_{A} /(\gamma-1)-((\gamma+1) /(\gamma-1))(C \sqrt{\gamma} / H) \beta^{(\gamma-1) / 2 \gamma}\right)(t-\tau) \\
-((\gamma+1) / 2) \beta(t-\tau)^{2} .
\end{array}
$$

One may check that for $\beta=\beta_{0}$ Eq. (7.6) is again obtained. Also it is clear that (7.9) represents a family of parabolae (parameter $\beta$ ). Each member passes through $x=0$, $t=\tau$.

Finally, the $r$-characteristics in the expansion region have to be considered. Employing the form (6.17) an expression for $1 / h$ in terms of $R^{*}, H$ and $(t-\tau)$ is easily found. Eliminating $h$, one is led to

$$
\begin{aligned}
& \frac{x}{t-\tau}=\left(U+2 a_{A} /(\gamma-1)\right)-((\gamma+1) / 2)(C / \sqrt{\gamma}) \\
& \cdot\left((2 \gamma /(\gamma-1)) H^{-1}+\left(R^{*} / H(t-\tau)\right)\right)\left((C / \sqrt{\gamma})\left(R^{*} /\left(H(t-\tau)^{2}\right)\right)\right)^{(\gamma-1) /(\gamma+1)} .
\end{aligned}
$$

The $r$-characteristics are essentially composed of three terms, each with a different power of $(t-\tau)$. The exponents of these three powers are respectively $1,(3-\gamma) /(\gamma-1)$ and $-2(\gamma-1) /(\gamma+1)$.

The formulae obtained in this section will take their simplest form if $\tau=0$ and $U=0$, but no attempt has yet been made to plot the $x, t$-plane in detail.

8. Final remarks. It was pointed out to me by Professor F. A. Pillow and later by Professor J. M. Burgers that Eq. (3.1) represents the pressure distribution in a gas 
at rest under the influence of gravity with $\beta_{0}=g$. Professor Burgers pursued this idea further and made the following observations: imagine a vertical column of gas at rest in the field of gravity, supported by a horizontal plane at $x=0$ and with the negative $x$-axes pointing upwards. The total mass of the gas is $H$ and the temperature increases in the upward direction in agreement with (3.3). The pressure distribution in the gas and the relation between $x$ and $h$ in the gas are given by (3.3) and (3.6) with $\beta_{0}=g$.

At time $t=\tau$ the supporting plane at $x=0$ is suddenly removed and the gas falls freely in the vacuum extending downwards over $0<x \leq+\infty$. The equations of motion are

$$
(\partial V / \partial t)-(\partial u / \partial h)=0, \quad(\partial u / \partial t)+(\partial p / \partial h)=g .
$$

When one writes

$$
u^{\prime}=u-g(t-\tau),
$$

they take the form

$$
(\partial V / \partial t)-\left(\partial u^{\prime} / \partial h\right)=0, \quad\left(\partial u^{\prime} / \partial t\right)+(\partial p / \partial h)=0 .
$$

Eqs. (8.3) and (8.4) are identical with the equations of motion used before and the answers obtained there immediately apply to the present problem, provided we replace $u$ by $u^{\prime}, \beta_{0}$ by $g$ and take $U=0$ at $t=\tau$.

Acknowledgements. The author would like to express his gratitude to the authorities in charge of the Visiting Netherlands Professorship at the University of Michigan, to his colleagues in Delft for taking over some of his duties during the academic year 1970-1971 and to his colleagues in Ann Arbor for their hospitality and for providing ideal working conditions. $\mathrm{He}$ is indebted to Professor J. M. Burgers and Professor F. A. Pillow for helpful comments.

\section{REFERENCES}

[1] G. S. S. Ludford and M. H. Martin, One-dimensional anisentropic flows, Comm. Pure Appl. Math. 7, 45-63 (1954)

[2] K. P. Stanyukovich, Unsteady moiion of continuous media (Transl. from Russian; M. Holt, editor), Academic Press, New York, 1960

[3] Ya. B. Zeldovich and Yu. P. Raizer, Physics of shock waves and high-temperature hydrodynamic phenomena (Transl. from Russian by W. D. Hayes and R. F. Probstein), Academic Press, New York, 1966

[4] D. G. Weir, A family of exact solutions of one-dimensional anisentropic flow, Proc. Cambr. Phil. Soc. 57, 890-894 (1961) 